\title{
Generalised Two-Component Modified Weakly Dissipative Dullin-Gottwald-Holm System: Invariance Analysis and Conservation Laws
}

\section{Sachin Kumar and Divya Jyoti}

\author{
Department of Mathematics and Statistics, School of Basic Sciences, Central \\ University of Punjab \\ Bathinda, Punjab, 151401 India \\ E-mail(corresp.): divya.jyoti1995@gmail.com \\ E-mail: sachin1jan@yahoo.com
}

Received January 19, 2021; revised December 14, 2021; accepted December 15, 2021

\begin{abstract}
The Dullin-Gottwald-Holm equation models the unidirectional propagation of shallow regime water waves. In this work, the Lie symmetry analysis of the generalised two-component modified weakly dissipative Dullin-Gottwald-Holm system is performed. Using symmetry reduction, the exact solutions are obtained in the form of power series and trigonometric functions. Also using multiplier approach, the conservation laws are obtained. The 3D graphical representations are also shown for obtained solutions.
\end{abstract}

Keywords: weakly dissipative Dullin-Gottwald-Holm system, Lie symmetries, exact solutions, conservation laws.

AMS Subject Classification: 35B06; 35C07; 35C09; 35C10; 35C11; 35G50.

\section{Introduction}

Dullin et al. [7] discussed the $1+1$ quadratically nonlinear equation

$$
u_{t}+\left(c_{0}+3 u\right) u_{x}+\beta u_{x x x}-\alpha^{2}\left(2 u_{x} u_{x x}+u u_{x x x}+u_{x x t}\right)=0, \quad x \in \mathbb{R}, t>0,
$$

known as the Dullin-Gottwald-Holm (DGH) equation. Here, $u$ represents the fluid velocit in $x$ direction; the constants $\frac{\beta}{c_{0}}$ and $\alpha^{2}$ are squares of length scales, and $c_{0}=\sqrt{g h}>0$ is the linear wave speed for undisturbed water at rest at spatial infinity where $g$ is the gravitational constant and $h$ is the mean

Copyright (C) 2022 The Author(s). Published by Vilnius Gediminas Technical University

This is an Open Access article distributed under the terms of the Creative Commons Attribution License (http://creativecommons.org/licenses/by/4.0/), which permits unrestricted use, distribution, and reproduction in any medium, provided the original author and source are credited. 
fluid depth. DGH equation models the unidirectional propagation of shallow water waves over a flat bottom. For $\alpha=0$, it becomes the Korteweg-de Vries equation; while for $\beta=0, \alpha=1,(1.1)$ recovers the well known Camassa-Holm $(\mathrm{CH})$ equation.

The $\mathrm{CH}$ equation arises while studying some non-Newtonian fluids. It models small amplitude, finite length radial deformation waves in hyperelastic rods. This completely integrable equation has bi-Hamiltonian structure and so, it possesses infinitely many conservation laws [2]. It's property of presence of breaking waves has attracted lot of attention [6]. For $\beta=0, \alpha=1, c_{0}=2 k$ ( $k$ an arbitrary constant), (1.1) recovers the Fuchssteiner-Fokas-Camassa-Holm equation, which has "peakon" solitary wave solution [5].

Lie group method $[1,19]$ is a powerful method to find the invariant solutions of system of nonlinear differential equations. This method is used for finding the symmetries, for symmetry reduction [15] and for finding the invariant solutions of system of nonlinear partial differential equations (PDEs) $[11,12,13,14]$. By using this method, the invariant solutions of the DGH equation have been found by Gupta and Anupma [9] as well as, the invariant solutions of the dissipative DGH equation have been found by Wei and Wang [24].

Guo et al. [8] followed the Ivanov's approach in the presence of a linear shear flow and non-zero vorticity to derive the following two-component DGH system $[3,4]$ as

$$
\begin{aligned}
& u_{t}+\left(c_{0}+3 u\right) u_{x}+\beta u_{x x x}-\alpha^{2}\left(2 u_{x} u_{x x}+u u_{x x x}+u_{x x t}\right)+\rho \rho_{x}=0, \\
& \rho_{t}+(\rho u)_{x}=0
\end{aligned}
$$

To allow the dependence on the average density $\bar{\rho}$ as well as the pointwise density $\rho$, this system (1.2) has been modified to modified two-component DGH system [25] as

$$
\begin{aligned}
& u_{t}+\left(c_{0}+3 u\right) u_{x}+\beta u_{x x x}-\alpha^{2}\left(2 u_{x} u_{x x}+u u_{x x x}+u_{x x t}\right)-\gamma \rho \overline{\rho_{x}}=0, \\
& \rho_{t}+(\rho u)_{x}=0 .
\end{aligned}
$$

Here, $\rho=\left(1-\partial_{x}^{2}\right)\left(\bar{\rho}-\overline{\rho_{0}}\right)$, with $\overline{\rho_{0}}$ to be constant and $\gamma$ is the downward acceleration of gravity in applications to shallow water waves.

It is important to include energy dissipation mechanisms in experiments for real waves. Therefore, Tian [21] considered weakly dissipative modified two-component DGH system (mDGH2) as

$$
\begin{aligned}
& u_{t}+\left(c_{0}+3 u\right) u_{x}+\beta u_{x x x}-\alpha^{2}\left(2 u_{x} u_{x x}+u u_{x x x}+u_{x x t}\right) \\
& \quad+\lambda\left(u-\alpha^{2} u_{x x}\right)-\gamma \rho \overline{\rho_{x}}=0 \\
& \rho_{t}+(\rho u)_{x}+\lambda \rho=0
\end{aligned}
$$

with weakly dissipative terms $\lambda\left(u-\alpha^{2} u_{x x}\right)$ and $\lambda \rho$, where $\lambda$ is a dissipative parameter. Tian derived asymptotic behaviour [21] and infinite propagation speed [22] of weakly dissipative mDGH2 system. The blow up phenomena of weakly dissipative mDGH2 system has also been derived by Tian et al. [23]. 
In this paper, weakly dissipative mDGH2 system is considered in a generalised form as

$$
\begin{aligned}
& u_{t}-\alpha^{2} u_{x x t}+\left(c_{0}+3 u\right) u_{x}+\beta u_{x x x}-\sigma \alpha^{2}\left(2 u_{x} u_{x x}+u u_{x x x}\right) \\
& \quad+\lambda\left(u-\alpha^{2} u_{x x}\right)-\gamma \rho \overline{\rho_{x}}=0 \\
& \quad \rho_{t}+(\rho u)_{x}+\lambda \rho=0
\end{aligned}
$$

This system includes several classical shallow water wave models. For example, if $\sigma=1$, this system becomes the weakly dissipative mDGH2 system.

Let $w=\bar{\rho}-\overline{\rho_{0}}$ in the above system (1.3), then it can be transformed as

$$
\begin{aligned}
& u_{t}-\alpha^{2} u_{x x t}+\left(c_{0}+3 u\right) u_{x}+\beta u_{x x x}-\sigma \alpha^{2}\left(2 u_{x} u_{x x}+u u_{x x x}\right) \\
& +\lambda\left(u-\alpha^{2} u_{x x}\right)-\gamma w_{x}\left(w-w_{x x}\right)=0, \\
& w_{t}-w_{x x t}+u\left(w_{x}-w_{x x x}\right)+u_{x}\left(w-w_{x x}\right)+\lambda\left(w-w_{x x}\right)=0 .
\end{aligned}
$$

The symmetries of generalised weakly dissipative mDGH2 system (1.4) are obtained by using Lie symmetry method. The exact solutions of system (1.4) are found in the form of power series [16] and other exact solutions in the form of hyperbolic and trigonometric functions. The separated and combined 3D plots are shown for the obtained solutions. The conservation laws [10] are also derived by using the multiplier approach $[17,18]$.

\section{Symmetry reduction}

The Lie classical method $[1,12,19]$ is used in this section for symmetry analysis in order to solve the above system of nonlinear PDEs (1.4).

Proposition 1. Equation (1.4) admits the following Lie symmetry operators:

$$
V_{1}=\partial_{x}, \quad V_{2}=\partial_{t}
$$

Proof. Consider the Lie group of point transformations

$$
\begin{array}{ll}
x^{*}=x+\epsilon \xi(x, t, u, w)+O\left(\epsilon^{2}\right), & t^{*}=t+\epsilon \tau(x, t, u, w)+O\left(\epsilon^{2}\right), \\
u^{*}=u+\epsilon \eta(x, t, u, w)+O\left(\epsilon^{2}\right), & w^{*}=w+\epsilon \zeta(x, t, u, w)+O\left(\epsilon^{2}\right),
\end{array}
$$

such that if $u, w$ satisfy (1.4), then $u^{*}, w^{*}$ also satisfy (1.4). By using the above group of transformations (2.1) in system of PDEs (1.4), the invariance conditions are

$$
\begin{aligned}
& \eta^{t}-\alpha^{2} \eta^{x x t}+\eta^{x}\left(c_{0}+3 u-2 \sigma \alpha^{2} u_{x x}\right)-\alpha^{2} \eta^{x x}\left(\lambda+2 \sigma u_{x}\right) \\
& \quad+\eta^{x x x}\left(\beta-\sigma \alpha^{2} u\right)+\eta\left(3 u_{x}+\lambda-\alpha^{2} \sigma u_{x x x}\right)=0 \\
& \zeta w_{x}-\zeta^{x x} w_{x}+\zeta^{x}\left(w-w_{x x}\right)=0 .
\end{aligned}
$$

By substituting the values of extended infinitesimals, and equating the coefficients of differentials of $u$ and $w$ to 0 , obtain a set of determining equations. 
The infinitesimals $\xi, \tau, \eta, \zeta$ obtained by solving the determining equations are found as

$$
\xi=C_{1}, \quad \tau=C_{2}, \quad \eta=0, \quad \zeta=0,
$$

where $C_{1}, C_{2}$ are arbitrary constants. The corresponding vector fields are $V_{1}=\partial_{x}, V_{2}=\partial_{t}$.

On considering the vector field $V_{2}+\epsilon V_{1}$, the similarity variables are obtained as

$$
r=x-\epsilon t, \quad u=g(r), \quad w=h(r),
$$

where $r$ is new independent variable and $g, h$ are new dependent functions. Back substituting these variables in equation (1.4), the reduced system of ODEs is obtained as

$$
\begin{aligned}
& \left(\alpha^{2} \epsilon+\beta-\sigma \alpha^{2} g\right) g^{\prime \prime \prime}-\alpha^{2}\left(2 \sigma g^{\prime}+\lambda\right) g^{\prime \prime}+\left(3 g+c_{0}-\epsilon\right) g^{\prime}+\lambda g+\gamma\left(h^{\prime \prime}-h\right) h^{\prime}=0 \\
& (\epsilon-g) h^{\prime \prime \prime}-\left(\lambda+g^{\prime}\right) h^{\prime \prime}+(g-\epsilon) h^{\prime}+\left(g^{\prime}+\lambda\right) h=0
\end{aligned}
$$

where' denotes the differentiation with respect to $r$.

\section{Exact solutions}

In this part, the exact solutions of system of ODEs (2.2) are obtained in the form of power series and other exact solutions in the form of hyperbolic and trigonometric functions.

\subsection{Power series solutions}

Let power series solution [16] be of the form

$$
g(r)=\sum_{m=0}^{\infty} a_{m} r^{m}, \quad h(r)=\sum_{m=0}^{\infty} b_{m} r^{m},
$$

for system of ODEs (2.2). Computing third order derivatives, product terms and substituting these computed values in (2.2), it can be expressed generally as

$$
\begin{aligned}
& -\sigma \alpha^{2} \sum_{m=1}^{\infty}\left(\sum_{k=0}^{m}(m-k+1)(m-k+2)(m-k+3) a_{k} a_{m-k+3}\right) r^{m}+3 a_{0} a_{1} \\
& +3 \sum_{m=1}^{\infty}\left(\sum_{k=0}^{m}(m-k+1) a_{k} a_{m-k+1}\right) r^{m}-\alpha^{2} \lambda \sum_{m=1}^{\infty}(m+1)(m+2) a_{m+2} r^{m} \\
& +\lambda a_{0}-2 \sigma \alpha^{2} \sum_{m=1}^{\infty}\left(\sum_{k=0}^{m}(k+1)(m-k+1)(m-k+2) a_{k+1} a_{m-k+2}\right) r^{m} \\
& +\left(c_{0}-\epsilon\right) \sum_{m=1}^{\infty}(m+1) a_{m+1} r^{m}+\left(\alpha^{2} \epsilon+\beta\right) \sum_{m=1}^{\infty}(m+1)(m+2)(m+3) a_{m+3} r^{m}
\end{aligned}
$$




$$
\begin{aligned}
& +6 a_{3}\left(\alpha^{2} \epsilon+\beta\right)+\gamma \sum_{m=1}^{\infty}\left(\sum_{k=0}^{m}(k+1)(m-k+1)(m-k+2) b_{k+1} b_{m-k+2}\right) r^{m} \\
& +2 b_{1} b_{2} \gamma-a_{0} a_{1} \gamma-\gamma \sum_{m=1}^{\infty}\left(\sum_{k=0}^{m}(m-k+1) b_{k} b_{m-k+1}\right) r^{m}+\left(c_{0}-\epsilon\right) a_{1} \\
& -6 \sigma \alpha^{2} a_{0} a_{3}+\lambda \sum_{m=1}^{\infty} a_{m} r^{m}-4 \sigma \alpha^{2} a_{1} a_{2}-2 a_{2} \alpha^{2} \lambda=0 \\
& -6 a_{0} b_{3}-\sum_{m=1}^{\infty}\left(\sum_{k=0}^{m}(m-k)\right. \\
& +\sum_{m=1}^{\infty}\left(\sum_{k=0}^{m}(m-k+1) a_{k} b_{m-k+1}\right) r^{m}-2 \lambda b_{2}-\lambda \sum_{m=1}^{\infty}(m+1)(m+2) b_{m+2} r^{m} \\
& -\sum_{m=1}^{\infty}\left(\sum_{k=0}^{m}(k+1)(m-k+1)(m-k+2) a_{k+1} b_{m-k+2}\right) r^{m} \\
& +\epsilon \sum_{m=1}^{\infty}(m+1)(m+2)(m+3) b_{m+3} r^{m}+\sum_{m=1}^{\infty}\left(\sum_{k=0}^{m}(m-k+1) b_{k} a_{m-k+1}\right) r^{m} \\
& +6 \epsilon b_{3}+a_{1} b_{0}-\epsilon b_{1}+\lambda b_{0}+\lambda \sum_{m=1}^{\infty} b_{m} r^{m}-2 a_{1} b_{2}-\epsilon \sum_{m=1}^{\infty}(m+1) b_{m+1} r^{m}=0 . a_{0} b_{1} \\
& +k+3) r^{m} \\
& +k+2 b)
\end{aligned}
$$

From above Equations (3.2a) and (3.2b) collecting coefficients of $r^{m}$, for $m=0$, it can be observed that

$$
\begin{aligned}
& a_{3}=\frac{\left(\lambda a_{0}+\left(c_{0}-\epsilon+3 a_{0}-a_{0} \gamma\right) a_{1}+2 b_{1} b_{2} \gamma-2 \alpha^{2} a_{2}\left(\lambda+2 a_{1} \sigma\right)\right)}{6 \alpha^{2}\left(a_{0} \sigma-\epsilon\right)-6 \beta} \\
& b_{3}=\frac{1}{6\left(a_{0}-\epsilon\right)}\left(\left(a_{0}-\epsilon\right) b_{1}-2\left(\lambda+a_{1}\right) b_{2}+\left(\lambda+a_{1}\right) b_{0}\right) .
\end{aligned}
$$

In general, for $m \geq 1$, it can be seen that

$$
\begin{aligned}
a_{m+3}= & \frac{1}{\left(\alpha^{2}\left(a_{0} \sigma-\epsilon\right)-\beta\right)(m+1)(m+2)(m+3)}\left(3 \sum_{k=0}^{m}(m-k+1) a_{k} a_{m-k+1}\right. \\
& -\sigma \alpha^{2} \sum_{k=1}^{m}(m-k+1)(m-k+2)(m-k+3) a_{k} a_{m-k+3}+\lambda a_{m} \\
& -2 \sigma \alpha^{2} \sum_{k=0}^{m}(k+1)(m-k+1)(m-k+2) a_{k+1} a_{m-k+2}
\end{aligned}
$$




$$
\begin{aligned}
& +\left(c_{0}-\epsilon\right)(m+1) a_{m+1}+\gamma \sum_{k=0}^{m}(k+1)(m-k+1)(m-k+2) b_{k+1} \\
& \left.\times b_{m-k+2}-\gamma \sum_{k=0}^{m}(m-k+1) b_{k} b_{m-k+1}-\alpha^{2} \lambda(m+1)(m+2) a_{m+2}\right) \\
& b_{m+3}=\frac{1}{\left(a_{0}-\epsilon\right)(m+1)(m+2)(m+3)}\left(-\sum_{k=0}^{m}(k+1)(m-k+1)(m-k+2)\right. \\
& \quad \times a_{k+1} b_{m-k+2}-\sum_{k=1}^{m}(m-k+1)(m-k+2)(m-k+3) a_{k} b_{m-k+3} \\
& +\sum_{k=0}^{m}(m-k+1) a_{k} b_{m-k+1}+\lambda b_{m}-\lambda(m+1)(m+2) b_{m+2} \\
& \left.-\epsilon(m+1) b_{m+1}+\sum_{k=0}^{m}(m-k+1) b_{k} a_{m-k+1}\right) .
\end{aligned}
$$

Thus, all the coefficients $a_{m}, b_{m}$, for $m \geq 4$ of power series (3.1) can be obtained from (3.3) and (3.4) as follows

$$
\begin{aligned}
a_{4}= & \frac{1}{24 \alpha^{2}\left(a_{0} \sigma-\epsilon\right)-24 \beta}\left(\lambda a_{1}-6 \alpha^{2}\left(3 \sigma a_{1} a_{3}+2 \sigma a_{2}^{2}+\lambda a_{3}\right)\right. \\
& \left.+2\left(c_{0}-\epsilon+3 a_{0}\right) a_{2}+3 a_{1}^{2}+\left(6 b_{1} b_{3}+4 b_{2}^{2}-2 b_{0} b_{2}-b_{1}^{2}\right) \gamma\right), \\
b_{4}= & \frac{1}{24\left(a_{0}-\epsilon\right)}\left(2\left(a_{0}-\epsilon\right) b_{2}-12 a_{1} b_{3}+2 a_{1} b_{1}+\lambda\left(b_{1}-6 b_{3}\right)+2\left(b_{0}-2 b_{2}\right) a_{2}\right), \\
a_{5}= & \frac{1}{60 \alpha^{2}\left(a_{0} \sigma-\epsilon\right)-60 \beta}\left(\lambda a_{2}-6 \alpha^{2}\left(8 \sigma a_{1} a_{4}+7 \sigma a_{2} a_{3}+2 \lambda a_{4}\right)\right. \\
& \left.+9 a_{1} a_{2}+3\left(c_{0}-\epsilon+3 a_{0}\right) a_{3}+3\left(6 b_{2} b_{3}-b_{1} b_{2}-b_{0} b_{3}+4 b_{1} b_{4}\right) \gamma\right), \\
b_{5}= & \frac{1}{60\left(a_{0}-\epsilon\right)}\left(3\left(a_{0}-\epsilon\right) b_{3}+3 a_{1}\left(b_{2}-12 b_{4}\right)+3 a_{2}\left(b_{1}-6 b_{3}\right)\right. \\
& \left.+\lambda\left(b_{2}-12 b_{4}\right)+3\left(b_{0}-2 b_{2}\right) a_{3}\right),
\end{aligned}
$$

and so on. Thus, for arbitrary chosen constants $a_{0}, a_{1}, a_{2}$ and $b_{0}, b_{1}, b_{2}$, the other terms of the sequences $\left\{a_{m}\right\}_{m=0}^{\infty}$ and $\left\{b_{m}\right\}_{m=0}^{\infty}$ can be determined successively from (3.3) and (3.4) in a unique manner. Therefore,

$$
\begin{aligned}
g(r) & =a_{0}+a_{1} r+a_{2} r^{2}+\frac{1}{6 \alpha^{2}\left(a_{0} \sigma-\epsilon\right)-6 \beta} \\
& \times\left(\lambda a_{0}+\left(c_{0}-\epsilon+3 a_{0}-a_{0} \gamma\right) a_{1}+2 b_{1} b_{2} \gamma-2 \alpha^{2} a_{2}\left(\lambda+2 a_{1} \sigma\right)\right) r^{3}+\ldots, \\
h(r) & =b_{0}+b_{1} r+b_{2} r^{2}+\frac{1}{6\left(a_{0}-\epsilon\right)}\left(\left(a_{0}-\epsilon\right) b_{1}-2\left(\lambda+a_{1}\right) b_{2}+\left(\lambda+a_{1}\right) b_{0}\right) r^{3}+\ldots
\end{aligned}
$$




\section{Convergence of power series}

Now we will check the convergence of the power series solution (3.1) of system of ODEs (2.2). From (3.4a), it can be observed that

$$
\begin{aligned}
\left|a_{m+3}\right| \leq & A\left(\sum_{k=0}^{m}\left|a_{k}\right|\left|a_{m-k+1}\right|+\left|a_{m}\right|+\sum_{k=1}^{m}\left|a_{k}\right|\left|a_{m-k+3}\right|+\left|a_{m+2}\right|\right. \\
& +\sum_{k=0}^{m}\left|a_{k+1}\right|\left|a_{m-k+2}\right|+\left|a_{m+1}\right|+\sum_{k=0}^{m}\left|b_{k+1}\right|\left|b_{m-k+2}\right| \\
& \left.+\sum_{k=0}^{m}\left|b_{k}\right|\left|b_{m-k+1}\right|\right), \quad m=1,2,3, \ldots,
\end{aligned}
$$

where

$$
\begin{aligned}
A=\max & \left\{\frac{|\lambda|}{\left|\alpha^{2}\left(a_{0} \sigma-\epsilon\right)-\beta\right|}, \frac{\left|2 \alpha^{2} \sigma\right|}{\left|\alpha^{2}\left(a_{0} \sigma-\epsilon\right)-\beta\right|}, \frac{\left|c_{0}-\epsilon\right|}{\left|\alpha^{2}\left(a_{0} \sigma-\epsilon\right)-\beta\right|},\right. \\
& \left.\frac{\left|\alpha^{2} \lambda\right|}{\left|\alpha^{2}\left(a_{0} \sigma-\epsilon\right)-\beta\right|}, \frac{|\gamma|}{\left|\alpha^{2}\left(a_{0} \sigma-\epsilon\right)-\beta\right|}, \frac{|3|}{\left|\alpha^{2}\left(a_{0} \sigma-\epsilon\right)-\beta\right|}\right\} .
\end{aligned}
$$

Similarly, from (3.4b), it can be observed that

$$
\begin{aligned}
\left|b_{m+3}\right| \leq & B\left(\sum_{k=0}^{m}\left|a_{k+1}\right|\left|b_{m-k+2}\right|+\sum_{k=1}^{m}\left|a_{k}\right|\left|b_{m-k+3}\right|+\sum_{k=0}^{m}\left|a_{k}\right|\left|b_{m-k+1}\right|\right. \\
& \left.+\left|b_{m+2}\right|+\left|b_{m+1}\right|+\sum_{k=0}^{m}\left|b_{k}\right|\left|a_{m-k+1}\right|\right), \quad m=1,2,3, \ldots
\end{aligned}
$$

where $B=\max \left\{|\lambda| /\left|a_{0}-\epsilon\right|,|\epsilon| /\left|a_{0}-\epsilon\right|,|1| /\left|a_{0}-\epsilon\right|\right\}$. Defining other power series as

$$
\mu=P(r)=\sum_{m=0}^{\infty} p_{m} r^{m}, \quad \nu=Q(r)=\sum_{m=0}^{\infty} q_{m} r^{m},
$$

such that $p_{i}=\left|a_{i}\right|$ and $q_{i}=\left|b_{i}\right|$ for $i=0,1,2,3$ and

$$
\begin{aligned}
p_{m+3}= & A\left(\sum_{k=0}^{m} p_{k} p_{m-k+1}+p_{m}+\sum_{k=1}^{m} p_{k} p_{m-k+3}+p_{m+2}+\sum_{k=0}^{m} p_{k+1} p_{m-k+2}\right. \\
& \left.+p_{m+1}+\sum_{k=0}^{m} q_{k+1} q_{m-k+2}+\sum_{k=0}^{m} q_{k} q_{m-k+1}\right), \\
q_{m+3}= & B\left(\sum_{k=0}^{m} p_{k+1} q_{m-k+2}+\sum_{k=1}^{m} p_{k} q_{m-k+3}+\sum_{k=0}^{m} p_{k} q_{m-k+1}+q_{m}\right. \\
& \left.+q_{m+2}+q_{m+1}+\sum_{k=0}^{m} q_{k} p_{m-k+1}\right),
\end{aligned}
$$

for $m=1,2,3, \ldots$. Then, it can be easily seen that $\left|a_{m}\right| \leq\left|p_{m}\right|,\left|b_{m}\right| \leq\left|q_{m}\right|$, for $m=0,1,2, \ldots$ Therefore, the series $\mu=P(r)=\sum_{m=0}^{\infty} p_{m} r^{m}$ and $\nu=Q(r)=$ $\sum_{m=0}^{\infty} q_{m} r^{m}$ are the majorant series of (3.1). 
Now, it remains to show that these series (3.5) have a positive radius of convergence. It can be calculated that

$$
\begin{aligned}
P(r) & =p_{0}+p_{1} r+p_{2} r^{2}+p_{3} r^{3}+\sum_{m=1}^{\infty} p_{m+3} r^{m+3} \\
= & p_{0}+p_{1} r+p_{2} r^{2}+p_{3} r^{3}+A\left(\sum_{m=1}^{\infty} \sum_{k=0}^{m} p_{k} p_{m-k+1} r^{m+3}+\sum_{m=1}^{\infty} p_{m} r^{m+3}\right. \\
& +\sum_{m=1}^{\infty} \sum_{k=1}^{m} p_{k} p_{m-k+3} r^{m+3}+\sum_{m=1}^{\infty} p_{m+2} r^{m+3}+\sum_{m=1}^{\infty} \sum_{k=0}^{m} p_{k+1} p_{m-k+2} r^{m+3} \\
& \left.+\sum_{m=1}^{\infty} p_{m+1} r^{m+3}+\sum_{m=1}^{\infty} \sum_{k=0}^{m} q_{k+1} q_{m-k+2} r^{m+3}+\sum_{m=1}^{\infty} \sum_{k=0}^{m} q_{k} q_{m-k+1} r^{m+3}\right) \\
= & p_{0}+p_{1} r+p_{2} r^{2}+p_{3} r^{3}+A\left(2 P^{2}-2 p_{0} P+Q^{2}-q_{0} Q\right. \\
& +\left(P-p_{0}-2 p_{0} p_{1}-q_{0} q_{1}\right) r+\left(P^{2}+P-p_{0}^{2}-p_{0}-p_{1}+Q^{2}-q_{0}^{2}\right) r^{2} \\
& +\left(-q_{1}^{2}-q_{0} q_{2}-2 p_{0} p_{2}-2 p_{1}^{2}-4 p_{0} p_{3}-4 p_{1} p_{2}\right) r^{2} \\
& \left.+\left(P-p_{0}+2 p_{0} p_{3}-2 p_{0} p_{1}-p_{1}-p_{2}-2 q_{0} q_{3}-2 q_{1} q_{2}-q_{0} q_{3}-2 q_{0} q_{1}\right) r^{3}\right),
\end{aligned}
$$

and

$$
\begin{aligned}
& Q(r)=q_{0}+q_{1} r+q_{2} r^{2}+q_{3} r^{3}+\sum_{m=1}^{\infty} q_{m+3} r^{m+3} \\
& =q_{0}+q_{1} r+q_{2} r^{2}+q_{3} r^{3}+B\left(\sum_{m=1}^{\infty} \sum_{k=0}^{m} p_{k+1} q_{m-k+2} r^{m+3}\right. \\
& \quad+\sum_{m=1}^{\infty} \sum_{k=0}^{m} p_{k} q_{m-k+1} r^{m+3}+\sum_{m=1}^{\infty} q_{m+2} r^{m+3}+\sum_{m=1}^{\infty} q_{m+1} r^{m+3} \\
& \left.+\sum_{m=1}^{\infty} \sum_{k=0}^{m} q_{k} p_{m-k+1} r^{m+3}+\sum_{m=1}^{\infty} \sum_{k=1}^{m} p_{k} q_{m-k+3} r^{m+3}+\sum_{m=1}^{\infty} q_{m} r^{m+3}\right) \\
& =q_{0}+q_{1} r+q_{2} r^{2}+q_{3} r^{3}+B\left(2 P Q-2 p_{0} Q+\left(Q-q_{0}-2 p_{1} q_{0}\right) r\right. \\
& \quad+\left(2 P Q+Q-q_{0}-q_{1}-2 q_{0} p_{2}-2 p_{1} q_{1}-2 p_{0} q_{0}\right) r^{2} \\
& \left.\quad+\left(Q-q_{0}-q_{1}-q_{2}-2 p_{0} q_{1}-2 q_{1} p_{2}-2 q_{0} p_{3}-2 q_{2} p_{1}-2 p_{1} q_{0}\right) r^{3}\right) .
\end{aligned}
$$

Now, considering the implicit functional equations in the form of independent variable $r$ as

$$
\begin{gathered}
F(r, \mu, \nu)=\mu-p_{0}-p_{1} r-p_{2} r^{2}-p_{3} r^{3}-A\left(2 \mu^{2}-2 p_{0} \mu+\nu^{2}-q_{0} \nu\right. \\
+\left(\mu-p_{0}-2 p_{0} p_{1}-q_{0} q_{1}\right) r+\left(\mu^{2}+\mu-p_{0}^{2}-p_{0}-p_{1}+\nu^{2}-q_{0}^{2}\right. \\
\left.\quad-q_{1}^{2}-q_{0} q_{2}-2 p_{0} p_{2}-2 p_{1}^{2}-4 p_{0} p_{3}-4 p_{1} p_{2}\right) r^{2}+\left(-p_{0}+2 p_{0} p_{3}\right) r^{3}
\end{gathered}
$$




$$
\begin{aligned}
& \left.+\left(\mu-2 p_{0} p_{1}-p_{1}-p_{2}-2 q_{0} q_{3}-2 q_{1} q_{2}-q_{0} q_{3}-2 q_{0} q_{1}\right) r^{3}\right) \\
G(r, \mu, \nu)= & \nu-q_{0}-q_{1} r-q_{2} r^{2}-q_{3} r^{3}-B\left(2 P Q-2 p_{0} Q+\left(Q-q_{0}-2 p_{1} q_{0}\right) r\right. \\
& +\left(2 P Q+Q-q_{0}-q_{1}-2 q_{0} p_{2}-2 p_{1} q_{1}-2 p_{0} q_{0}\right) r^{2} \\
& \left.+\left(Q-q_{0}-q_{1}-q_{2}-2 p_{0} q_{1}-2 q_{1} p_{2}-2 q_{0} p_{3}-2 q_{2} p_{1}-2 p_{1} q_{0}\right) r^{3}\right) .
\end{aligned}
$$

Since $F(r, \mu, \nu)$ and $G(r, \mu, \nu)$ are analytic in the neighborhood of $\left(0, p_{0}, q_{0}\right)$ and $F\left(0, p_{0}, q_{0}\right)=0, G\left(0, p_{0}, q_{0}\right)=0$. Furthermore, the Jacobian determinant

$$
J=\frac{\partial(F, G)}{\partial(\mu, \nu)}=1-2 A p_{0}-2 A B q_{0}^{2} \neq 0,
$$

if the parameters $p_{0}=\left|a_{0}\right|$ and $q_{0}=\left|b_{0}\right|$ are chosen suitably. By the implicit function theorem [20], it can be seen that $\mu=P(r)$ and $\nu=Q(r)$ are analytic in a neighborhood of point $\left(0, p_{0}, q_{0}\right)$. This shows that both the power series (3.1) converge in a neighborhood of point $\left(0, p_{0}, q_{0}\right)$. This completes the proof. Thus, the exact analytic power series solution of generalised weakly dissipative mDGH2 system (1.4) can be written as

$$
\begin{aligned}
& u(x, t)=a_{0}+a_{1}(x-\epsilon t)+a_{2}(x-\epsilon t)^{2}+\frac{1}{6 \alpha^{2}\left(a_{0} \sigma-\epsilon\right)-6 \beta} \\
& \times\left(\lambda a_{0}+\left(c_{0}-\epsilon+3 a_{0}-a_{0} \gamma\right) a_{1}+2 b_{1} b_{2} \gamma-2 \alpha^{2} a_{2}\left(\lambda+2 a_{1} \sigma\right)\right) \\
& \times(x-\epsilon t)^{3}+\sum_{m=1}^{\infty} a_{m+3}(x-\epsilon t)^{m+3}, \\
& w(x, t)=b_{0}+b_{1}(x-\epsilon t)+b_{2}(x-\epsilon t)^{2}+\left(\left(a_{0}-\epsilon\right) b_{1}-2\left(\lambda+a_{1}\right) b_{2}+\left(\lambda+a_{1}\right) b_{0}\right) \\
& \quad \times \frac{(x-\epsilon t)^{3}}{6\left(a_{0}-\epsilon\right)}+\sum_{m=1}^{\infty} b_{m+3}(x-\epsilon t)^{m+3},
\end{aligned}
$$

where $a_{m+3}$ and $b_{m+3}$ are given by recurrence relation (3.4).

\subsection{Travelling wave solutions}

In order to obtain travelling wave solutions [9] of the weakly dissipative mDGH2 system (1.4), the different particular values of parameters of ODE system (2.2) are considered.

\subsection{1 $\alpha= \pm \frac{1}{3}, c_{0}=-9 \beta, \sigma=1$}

For $\alpha= \pm \frac{1}{3}, c_{0}=-9 \beta, \sigma=1$ and keeping all other parameters same, the ODE system (2.2) reduces to

$$
\begin{aligned}
& (\epsilon+9 \beta-g) g^{\prime \prime \prime}-\left(2 g^{\prime}+\lambda\right) g^{\prime \prime}+9(3 g-9 \beta-\epsilon) g^{\prime}+9 \lambda g+9 \gamma\left(h^{\prime \prime}-h\right) h^{\prime}=0, \\
& (\epsilon-g) h^{\prime \prime \prime}-\left(\lambda+g^{\prime}\right) h^{\prime \prime}+(g-\epsilon) h^{\prime}+\left(g^{\prime}+\lambda\right) h=0 .
\end{aligned}
$$

Equation (3.7) possesses travelling wave solutions as

$$
\begin{aligned}
& g(r)=C_{3} \iota \sinh (r)\left(4 \cosh ^{2}(r)-1\right), \quad h(r)=C_{4} \cosh (r), \\
& g(r)=C_{7} e^{ \pm 3 r+3 C_{6}}, \quad h(r)=C_{5} e^{ \pm r+C_{6}}
\end{aligned}
$$


where $\iota$ represents an imaginary number, $C_{3}, C_{4}, C_{5}, C_{6}$ and $C_{7}$ are arbitrary constants. Thus, the travelling wave solutions of the mDGH2 system (1.4) are

$$
\begin{aligned}
& u(x, t)=C_{3} \iota \sinh (x-\epsilon t)\left(4 \cosh ^{2}(x-\epsilon t)-1\right), \\
& w(x, t)=C_{4} \cosh (x-\epsilon t), \\
& u(x, t)=C_{7} e^{ \pm 3(x-\epsilon t)+3 C_{6}}, \quad w(x, t)=C_{5} e^{ \pm(x-\epsilon t)+C_{6}} .
\end{aligned}
$$

\subsection{2 $\alpha= \pm 1, \beta=-c_{0}, \sigma=1$}

For $\alpha= \pm 1, \beta=-c_{0}, \sigma=1$ and keeping all other parameters same, the ODE system $(2.2)$ reduces to

$$
\begin{aligned}
& \left(\epsilon-c_{0}-g\right) g^{\prime \prime \prime}-\left(2 g^{\prime}+\lambda\right) g^{\prime \prime}+\left(3 g+c_{0}-\epsilon\right) g^{\prime}+\lambda g+\gamma\left(h^{\prime \prime}-h\right) h^{\prime}=0, \\
& (\epsilon-g) h^{\prime \prime \prime}-\left(\lambda+g^{\prime}\right) h^{\prime \prime}+(g-\epsilon) h^{\prime}+\left(g^{\prime}+\lambda\right) h=0 .
\end{aligned}
$$

Equation (3.9) possesses travelling wave solutions as

$$
\begin{aligned}
& g(r)=C_{3} \cosh ^{3}\left(\frac{r}{3}\right)-\frac{3}{4} C_{3} \cosh \left(\frac{r}{3}\right) \\
& h(r)=C_{4} \iota \sinh \left( \pm \frac{r}{3}\right)\left(4 \cosh ^{2}\left(\frac{r}{3}\right)-1\right) \\
& g(r)=C_{6} \sinh ^{3}\left( \pm \frac{1}{3} r+C_{7}\right)+\frac{3}{4} C_{6} \sinh \left( \pm \frac{1}{3} r+C_{7}\right) \\
& h(r)=C_{5} \sinh ^{3}\left( \pm \frac{1}{3} r+C_{7}\right)+\frac{3}{4} C_{5} \sinh \left( \pm \frac{1}{3} r+C_{7}\right),
\end{aligned}
$$

where $C_{3}, C_{4}, C_{5}, C_{6}$ and $C_{7}$ are arbitrary constants. Thus, the travelling wave solutions of the mDGH2 system (1.4) are

$$
\begin{aligned}
\text { (i) } u(x, t) & =C_{3} \cosh ^{3}\left(\frac{x-\epsilon t}{3}\right)-\frac{3}{4} C_{3} \cosh \left(\frac{x-\epsilon t}{3}\right), \\
w(x, t) & =C_{4} \iota \sinh \left( \pm \frac{(x-\epsilon t)}{3}\right)\left(4 \cosh ^{2}\left(\frac{x-\epsilon t}{3}\right)-1\right) \\
\text { (ii) } u(x, t) & =C_{6} \sinh ^{3}\left( \pm \frac{(x-\epsilon t)}{3}+C_{7}\right)+\frac{3}{4} C_{6} \sinh \left( \pm \frac{1}{3}(x-\epsilon t)+C_{7}\right), \\
w(x, t) & =C_{5} \sinh ^{3}\left( \pm \frac{(x-\epsilon t)}{3}+C_{7}\right)+\frac{3}{4} C_{5} \sinh \left( \pm \frac{1}{3}(x-\epsilon t)+C_{7}\right) .
\end{aligned}
$$

$$
\text { 3.2.3 } \alpha= \pm \frac{3}{2}, \beta=-\frac{9}{4} c_{0}, \sigma=1
$$

For $\alpha= \pm \frac{3}{2}, \beta=-\frac{9}{4} c_{0}, \sigma=1$ and keeping all other parameters same, the ODE system (2.2) reduces to

$$
\begin{aligned}
& \frac{9}{4}\left(\epsilon-c_{0}-g\right) g^{\prime \prime \prime}-\frac{9}{4}\left(2 g^{\prime}+\lambda\right) g^{\prime \prime}+\left(3 g+c_{0}-\epsilon\right) g^{\prime}+\lambda g+\gamma\left(h^{\prime \prime}-h\right) h^{\prime}=0, \\
& (\epsilon-g) h^{\prime \prime \prime}-\left(\lambda+g^{\prime}\right) h^{\prime \prime}+(g-\epsilon) h^{\prime}+\left(g^{\prime}+\lambda\right) h=0 .
\end{aligned}
$$


Equation (3.11) possesses travelling wave solutions as

$$
\begin{aligned}
& g(r)=C_{3} \sinh ^{2}\left( \pm \frac{1}{3} r+C_{2}\right)+\frac{1}{2} C_{3} \\
& h(r)=C_{4} \sinh ^{3}\left( \pm \frac{1}{3} r+C_{2}\right)+\frac{3}{4} C_{4} \sinh \left( \pm \frac{1}{3} r+C_{2}\right)
\end{aligned}
$$

where $C_{2}, C_{3}$ and $C_{4}$ are arbitrary constants. Thus, the travelling wave solutions of the mDGH2 system (1.4) are

$$
\begin{aligned}
& u(x, t)=C_{3} \sinh ^{2}\left( \pm \frac{1}{3}(x-\epsilon t)+C_{2}\right)+\frac{1}{2} C_{3} \\
& w(x, t)=C_{4} \sinh ^{3}\left( \pm \frac{(x-\epsilon t)}{3}+C_{2}\right)+\frac{3}{4} C_{4} \sinh \left( \pm \frac{(x-\epsilon t)}{3}+C_{2}\right) .
\end{aligned}
$$

\subsection{4 $\alpha= \pm 3, \beta=-9 c_{0}, \sigma=1$}

For $\alpha= \pm 3, \beta=-9 c_{0}, \sigma=1$ and keeping all other parameters same, the ODE system (2.2) reduces to

$$
\begin{aligned}
& 9\left(\epsilon-c_{0}-g\right) g^{\prime \prime \prime}-9\left(2 g^{\prime}+\lambda\right) g^{\prime \prime}+\left(3 g+c_{0}-\epsilon\right) g^{\prime}+\lambda g+\gamma\left(h^{\prime \prime}-h\right) h^{\prime}=0 \\
& (\epsilon-g) h^{\prime \prime \prime}-\left(\lambda+g^{\prime}\right) h^{\prime \prime}+(g-\epsilon) h^{\prime}+\left(g^{\prime}+\lambda\right) h=0
\end{aligned}
$$

Equation (3.13) possesses travelling wave solutions as

$$
\begin{aligned}
& g(r)=C_{3} \cosh \left( \pm \frac{1}{3} r+C_{2}\right) \\
& h(r)=C_{4} \cosh ^{3}\left( \pm \frac{1}{3} r+C_{2}\right)-\frac{3}{4} C_{4} \cosh \left( \pm \frac{1}{3} r+C_{2}\right)
\end{aligned}
$$

where $C_{2}, C_{3}$ and $C_{4}$ are arbitrary constants. Thus, the travelling wave solutions of the mDGH2 system (1.4) are

$$
\begin{aligned}
& u(x, t)=C_{3} \cosh \left( \pm \frac{1}{3}(x-\epsilon t)+C_{2}\right) \\
& w(x, t)=C_{4} \cosh ^{3}\left( \pm \frac{(x-\epsilon t)}{3}+C_{2}\right)-\frac{3}{4} C_{4} \cosh \left( \pm \frac{(x-\epsilon t)}{3}+C_{2}\right) .
\end{aligned}
$$

\section{Conservation laws}

In this part, the local conservation laws [10] of generalised weakly dissipative mDGH2 system (1.4) are obtained by using the multiplier approach $[17,18]$. Consider the multipliers of the form

$$
\Lambda\left(x, t, u, w, u_{x}, w_{x}, u_{x x}, w_{x x}\right), q u a d \psi\left(x, t, u, w, u_{x}, w_{x}, u_{x x}, w_{x x}\right) .
$$


The simplified determining equations to be solved are

$$
\begin{aligned}
& \Lambda_{t}=-\frac{1}{\gamma}\left(\left(u \psi_{w}-\gamma \Lambda\right) \lambda\right), \quad \Lambda_{u}=-\frac{\psi_{w}}{\gamma}, \quad \psi_{t}=\psi_{w} \lambda w+\lambda \psi, \quad \psi_{w w}=0 \\
& \Lambda_{u_{x x}}=0, \quad \Lambda_{x}=0, \quad \Lambda_{u_{x}}=0, \quad \Lambda_{w_{x x}}=0, \quad \Lambda_{w}=0, \quad \Lambda_{w_{x}}=0 \\
& \psi_{u_{x x}}=0, \quad \psi_{x}=0, \quad \psi_{u_{x}}=0, \quad \psi_{w_{x x}}=0, \quad \psi_{u}=0, \quad \psi_{w_{x}}=0
\end{aligned}
$$

The solution of above determining equations (4.1) yields three multipliers as

$$
\begin{aligned}
& \Lambda^{(1)}=-\frac{u}{\gamma} e^{2 \lambda t}, \quad \psi^{(1)}=w e^{2 \lambda t}, \quad \Lambda^{(2)}=0, \quad \psi^{(2)}=e^{\lambda t}, \\
& \Lambda^{(3)}=e^{\lambda t}, \quad \psi^{(3)}=0 .
\end{aligned}
$$

Thus, the conserved fluxes in accordance to each multiplier (4.2) are

$$
\begin{aligned}
T_{t}^{(1)}= & \frac{e^{2 \lambda t}}{2 \gamma}\left(-u^{2}+\alpha^{2} u u_{x x}+\gamma w^{2}-\gamma w w_{x x}\right), \\
T_{x}^{(1)}= & \frac{e^{2 \lambda t}}{2 \gamma}\left(\alpha^{2} \sigma\left(2 u^{2} u_{x x}+u u_{x t}-u_{t} u_{x}\right)+\beta\left(u_{x}^{2}-2 u u_{x x}-u^{2}\left(c_{0}+2 u\right)\right)\right. \\
& \left.\quad+\gamma\left(2 u w^{2}-2 u w w_{x x}+w_{x} w_{t}-w w_{x t}\right)\right), \\
T_{t}^{(2)}= & e^{\lambda t}\left(w-w_{x x}\right), \quad T_{x}^{(2)}=u e^{\lambda t}\left(w-w_{x x}\right), \quad T_{t}^{(3)}=e^{\lambda t}\left(u-\alpha^{2} u_{x x}\right), \\
T_{x}^{(3)}= & -\frac{e^{\lambda t}}{2}\left(\alpha^{2} \sigma\left(2 u u_{x x}+u_{x}^{2}\right)+\gamma\left(w^{2}-w_{x}^{2}\right)-2 \beta u_{x x}-u\left(2 c_{0}+3 u\right)\right) .
\end{aligned}
$$

\section{Discussion}

The solution (3.12) contains arbitrary constants $C_{2}, C_{3}, C_{4}, \epsilon$ which lead to many possible solutions. The graphical representations are shown by considering a possibility of these constants.

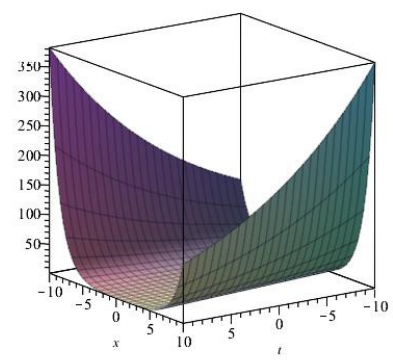

Figure 1. 3D plot of $u(x, t)$ of solution (3.12) by considering $C_{2}=0, C_{3}=1, C_{4}=-\frac{1}{5}, \epsilon=\frac{1}{10}$ and positive sign for angle.

Figures 1 and 2 depict the 3D graphs of solution (3.12) of (1.4) by considering $C_{2}=0, C_{3}=1, C_{4}=-\frac{1}{5}, \epsilon=\frac{1}{10}$ and positive sign for angle. The solution 


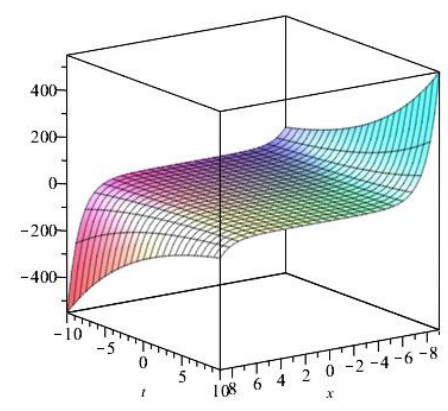

Figure 2. 3D plot of $w(x, t)$ of solution (3.12) by considering $C_{2}=0, C_{3}=1, C_{4}=-\frac{1}{5}, \epsilon=\frac{1}{10}$ and positive sign for angle.

(3.14) also contains arbitrary constants $C_{2}, C_{3}, C_{4}, \epsilon$ leading to infinite possible solutions. The graphical representations are shown by considering a possibility of these constants. Figures 3 and 4 depict the 3D graphs of solution (3.14) of (1.4) by considering $C_{2}=0, C_{3}=-13, C_{4}=1, \epsilon=\frac{1}{100}$ and positive sign for angle. The relation of solutions $u(x, t)$ and $w(x, t)$ can also be observed from

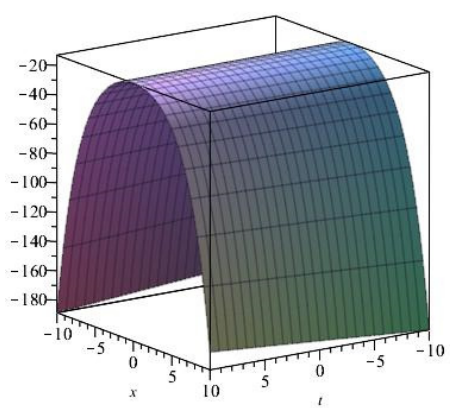

Figure 3. 3D plot of $u(x, t)$ of solution (3.14) by considering $C_{2}=0, C_{3}=-13, C_{4}=1, \epsilon=\frac{1}{100}$ and positive sign for angle.

their combined 3D plot as in Figure 5. The overlapping and non-overlapping parts, the smoothness, non-singural regions of $u(x, t)$ and $w(x, t)$ can be examined easily, due to their different shading. The lining shade is used for $u(x, t)$ while plain shade is used for $w(x, t)$. Figure 5 represents combined solution (3.14) by considering $C_{2}=0, C_{3}=10, C_{4}=-\frac{1}{12}, \epsilon=\frac{1}{50}$ and positive sign for angle. For $w(x, t)=0, \lambda=0, \sigma=1$, the solutions (3.6), (3.8), (3.10), (3.12), (3.14), are the new solutions of the DGH equation considered by Gupta and Anupma [9].

For $w(x, t)=0, \alpha=\sigma=1, \beta=-c_{0}$, the solutions (3.6), (3.10), are the new solutions of the dissipative DGH equation considered by Wei and Wang [24]. 


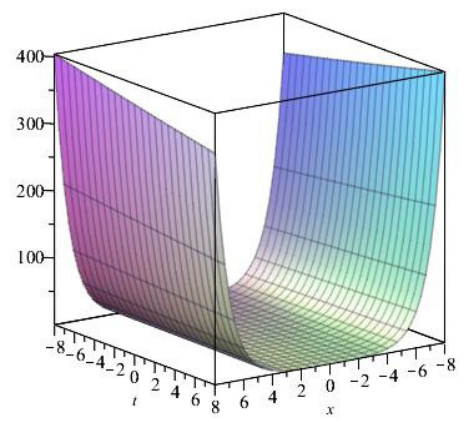

Figure 4. 3D plot of $w(x, t)$ of solution (3.14) by considering $C_{2}=0, C_{3}=-13, C_{4}=1$, $\epsilon=\frac{1}{100}$ and positive sign for angle.

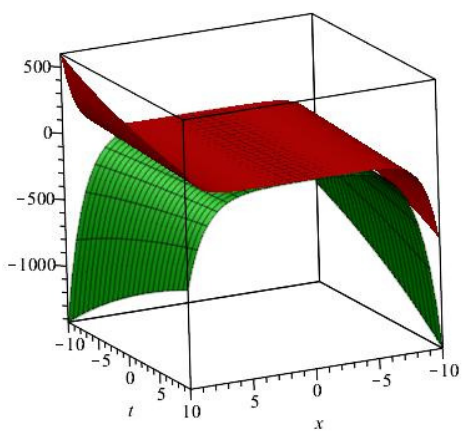

Figure 5. 3D plot of combined solution (3.14) by considering $C_{2}=0, C_{3}=10$, $C_{4}=-\frac{1}{12}, \epsilon=\frac{1}{50}$, positive sign for angle. Here, lining shade is used for $u(x, t)$ (upper) while plain shade is used for $w(x, t)$ (lower).

\section{Conclusions}

In this work, the Lie symmetry analysis of the generalised weakly dissipative modified two-component Dullin-Gottwald-Holm system has been performed. This system has been reduced to a system of ODEs by using the classical symmetries. Using symmetry reduction, the exact solutions of this system have been obtained in the form of power series (3.6) and other exact solutions in the form of hyperbolic and trigonometric functions (3.8),(3.10),(3.12),(3.14). The periodic solutions of this system has been found for the first time, to the best of author's knowledge. Also using multiplier approach, the conservation laws have been obtained. The 3D graphical representations have also been shown for obtained solutions. 


\section{Acknowledgements}

Second author (Divya Jyoti) is very much thankful to CSIR for providing financial assistance in terms of JRF fellowship via letter with Sr. No. 1061841352 and Ref. No. 17/06/2018(i)EU-V.

\section{References}

[1] G. Bluman and S. Anco. Symmetry and integration methods for differential equations, volume 154. Springer, New York, 2002.

[2] R. Camassa and D.D. Holm. An integrable shallow water equation with peaked solitons. Physical review letters, 71(11):1661-1664, 1993. https://doi.org/10.1103/PhysRevLett.71.1661.

[3] W. Cheng and T. Xu. Blow-up of solutions to a modified two-component Dullin-Gottwald-Holm system. Applied Mathematics Letters, 105:106307, 2020. https://doi.org/10.1016/j.aml.2020.106307.

[4] W. Cheng and T. Xu. Local-in-space blow-up and symmetry of traveling wave solutions to a generalized two-component Dullin-Gottwald-Holm system. Monatshefte für Mathematik, 193(3):573-589, 2020. https://doi.org/10.1007/s00605020-01411-w.

[5] P.A. Clarkson, E.L. Mansfield and T.J. Priestley. Symmetries of a class of nonlinear third-order partial differential equations. Mathematical and Computer Modelling, 25(8-9):195-212, 1997. https://doi.org/10.1016/S0895-7177(97)00069-1.

[6] A. Constantin and R.I. Ivanov. On an integrable two-component CamassaHolm shallow water system. Physics Letters A, 372(48):7129-7132, 2008. https://doi.org/10.1016/j.physleta.2008.10.050.

[7] H.R. Dullin, G.A. Gottwald and D.D. Holm. An integrable shallow water equation with linear and nonlinear dispersion. Phys. Rev. Lett., 87(19):194501, 2001. https://doi.org/10.1103/PhysRevLett.87.194501.

[8] F. Guo, H. Gao and Y. Liu. On the wave-breaking phenomena for the twocomponent Dullin-Gottwald-Holm system. J. London Math. Soc., 86(3):810834, 2012. https://doi.org/10.1112/jlms/jds035.

[9] R.K. Gupta and Anupma. The Dullin-Gottwald-Holm equation: Classical Lie approach and exact solutions. Int. J. Nonlinear Sci., 10(2):146-152, 2010. ISSN 1749-3889.

[10] N.H. Ibragimov. A new conservation theorem. Journal of Mathematical Analysis and Applications, $333(1): 311-328, \quad 2007$. https://doi.org/10.1016/j.jmaa.2006.10.078.

[11] D. Jyoti and S. Kumar. Exact non-static solutions of Einstein vacuum field equations. Chinese Journal of Physics, 68:735-744, 2020. https://doi.org/10.1016/j.cjph.2020.10.006.

[12] D. Jyoti and S. Kumar. Modified Vakhnenko-Parkes equation with power law nonlinearity: Painlevé analysis, analytic solutions and conservation laws. The European Physical Journal Plus, 135:762, 2020. https://doi.org/10.1140/epjp/s13360-020-00785-y.

[13] D. Jyoti and S. Kumar. Invariant solutions and conservation laws of Einstein field equations in non-comoving radiation fields. Chinese Journal of Physics, 70:37-43, 2021. https://doi.org/10.1016/j.cjph.2020.12.018. 
[14] D. Jyoti, S. Kumar and R.K. Gupta. Exact solutions of Einstein field equations in perfect fluid distribution using Lie symmetry method. The European Physical Journal Plus, 135:604,2020. https://doi.org/10.1140/epjp/s13360-020-00622-2.

[15] S. Kumar. Invariant solutions of Biswas-Milovic equation. Nonlinear Dyn., 87(2):1153-1157, 2016. https://doi.org/10.1007/s11071-016-3105-6.

[16] H. Liu and Y. Geng. Symmetry reductions and exact solutions to the systems of carbon nanotubes conveying fluid. J. Differ. Equations, 254(5):2289-2303, 2013. https://doi.org/10.1016/j.jde.2012.12.004.

[17] R. Naz. Conservation laws for some systems of nonlinear partial differential equations via multiplier approach. J. Appl. Math., 2012:871253, 2012. https://doi.org/10.1155/2012/871253.

[18] R. Naz, I. Naeem and M. Khan. Conservation laws of some physical models via symbolic package GeM. Math. Probl. Eng., 2013:897912, 2013. https://doi.org/10.1155/2013/897912.

[19] P.J. Olver. Applications of Lie groups to differential equations. Springer, New York, 1986. https://doi.org/10.1007/978-1-4684-0274-2.

[20] W. Rudin. Principles of mathematical analysis, volume 3. McGraw-hill, New York, 1964.

[21] S.F. Tian. Asymptotic behavior of a weakly dissipative modified twocomponent Dullin-Gottwald-Holm system. Appl. Math. Lett., 83:65-72, 2018. https://doi.org/10.1016/j.aml.2018.03.019.

[22] S.F. Tian. Infinite propagation speed of a weakly dissipative modified twocomponent Dullin-Gottwald-Holm system. Appl. Math. Lett., 89:1-7, 2019. https://doi.org/10.1016/j.aml.2018.09.010.

[23] S.F. Tian, J.J. Yang, Z.Q. Li and Y.R. Chen. Blow-up phenomena of a weakly dissipative modified two-component Dullin-Gottwald-Holm system. Appl. Math. Lett., 106:106378, 2020. https://doi.org/10.1016/j.aml.2020.106378.

[24] L. Wei and Y. Wang. Symmetry analysis, conserved quantities and applications to a dissipative DGH equation. Journal of Differential Equations, 266(6):31893208, 2019. https://doi.org/10.1016/j.jde.2018.08.055.

[25] P. Zhai, Z. Guo and W. Wang. Wave breaking phenomenon for a modified twocomponent Dullin-Gottwald-Holm equation. J. Math. Phys., 55(9):093101, 2014. https://doi.org/10.1063/1.4894368. 